\title{
Physical and magnetic properties of $\mathrm{Ba}\left(\mathrm{Fe}_{1-x} \mathrm{Ru}_{x}\right)_{2} \mathrm{As}_{2}$ single crystals
}

\author{
A.Thaler, N. Ni, A. Kracher, J. Q. Yan, S.L. Bud'ko, and P.C. Canfield \\ Ames Laboratory and Department of Physics and Astronomy, \\ Iowa State University, Ames, IA 50011, USA
}

(Dated: November 8, 2018)

\begin{abstract}
Single crystals of $\mathrm{Ba}\left(\mathrm{Fe}_{1-x} \mathrm{Ru}_{x}\right)_{2} \mathrm{As}_{2}, x<0.37$, have been grown and characterized by structural, magnetic and transport measurements. These measurements show that the structural/magnetic phase transition found in pure $\mathrm{BaFe}_{2} \mathrm{As}_{2}$ at $134 \mathrm{~K}$ is suppressed monotonically by Ru doping, but, unlike doping with $\mathrm{TM}=\mathrm{Co}, \mathrm{Ni}, \mathrm{Cu}, \mathrm{Rh}$ or $\mathrm{Pd}$, the coupled transition seen in the parent compound does not detectably split into two separate ones. Superconductivity is stabilized at low temperatures for $x>0.2$ and continues through the highest doping levels we report. The superconducting region is dome like, with maximum $\mathrm{T}_{c}(\sim 16.5 \mathrm{~K})$ found around $x \sim 0.29$. A phase diagram of temperature versus doping, based on electrical transport and magnetization measurements, has been constructed and compared to those of the $\mathrm{Ba}\left(\mathrm{Fe}_{1-x} \mathrm{TM}_{x}\right)_{2} \mathrm{As}_{2}(\mathrm{TM}=\mathrm{Co}, \mathrm{Ni}, \mathrm{Rh}, \mathrm{Pd})$ series as well as to the temperature-pressure phase diagram for pure $\mathrm{BaFe}_{2} \mathrm{As}_{2}$. Suppression of the structural/magnetic phase transition as well as the appearance of superconductivity is much more gradual in $\mathrm{Ru}$ doping, as compared to $\mathrm{Co}, \mathrm{Ni}, \mathrm{Rh}$ and $\mathrm{Pd}$ doping, and appears to have more in common with $\mathrm{BaFe}_{2} \mathrm{As}_{2}$ tuned with pressure; by plotting $T_{S} / T_{m}$ and $T_{c}$ as a function of changes in unit cell dimensions, we find that changes in the $c / a$ ratio, rather than changes in $c, a$ or $\mathrm{V}$, unify the $T(p)$ and $T(x)$ phase diagrams for $\mathrm{BaFe}_{2} \mathrm{As}_{2}$ and $\mathrm{Ba}\left(\mathrm{Fe}_{1-x} \mathrm{Ru}_{x}\right)_{2} \mathrm{As}_{2}$ respectively.

PACS numbers: 74.10.+v, 74.25.Dw, 74.62.Bf, 74.62.Dh, 74.70.Xa
\end{abstract}




\section{INTRODUCTION}

The discovery of superconductivity in F-doped $\mathrm{LaFeAsO}[1]$ and $\mathrm{K}$-doped $\mathrm{BaFe}_{2} \mathrm{As}_{2}[2]$ in 2008 led to extensive interest in these families of FeAs-based compounds. The superconducting critical temperature, $T_{c}$, has risen as high as $56 \mathrm{~K}$ for $\mathrm{F}$ doped $\mathrm{RFeAsO}[3$ ] and as high as $38 \mathrm{~K}$ in $\mathrm{K}$ and $\mathrm{Na}$ doped $\mathrm{AEFe}_{2} \mathrm{As}_{2}$ systems (AE=Ba, Sr, Ca) 2]. Superconductivity was also found in $\mathrm{Co}$ doped $\mathrm{AEFe}_{2} \mathrm{As}_{2}$ [4] and RFeAsO [5]. More recently, superconductivity has been found in other $3 \mathrm{~d}, 4 d$ and $5 d$ transition metal, electron doped $\mathrm{BaFe}_{2} \mathrm{As}_{2}$ systems [6-14], as well as $\mathrm{SrFe}_{2} \mathrm{As}_{2}$ and $\mathrm{CaFe}_{2} \mathrm{As}_{2}$. Although the electron doped $\mathrm{AEFe}_{2} \mathrm{As}_{2}$ systems have lower $T_{c}$ values than the hole doped ones [10 14], they have been studied extensively because

doping is more homogeneous in these systems and single crystals can be more easily and reproduceably grown. In order to understand the conditions for superconductivity in these systems, temperature versus doping phase diagrams must first be constructed. Detailed studies have been made for TM doped $\mathrm{BaFe}_{2} \mathrm{As}_{2}(\mathrm{TM}=\mathrm{Co}, \mathrm{Ni}, \mathrm{Cu}, \mathrm{Rh}, \mathrm{Pd}, \mathrm{Pt}, \mathrm{Ir})$ [6, 1016]. For $\mathrm{Co}, \mathrm{Ni}, \mathrm{Cu}, \mathrm{Rh}$ and $\mathrm{Pd}$, temperature vs doping concentration, $x$, and temperature vs electron count, $e$, phase diagrams show similar properties, with the temperature of the structural/magnetic transition, $T_{S} / T_{m}$, seen in the parent compound being suppressed and separated in a similar manner with $x$, and $T_{c}$ evolving in a similar manner with $e$, especially on the overdoped side of the superconducting dome [6, 10, 14, 17]. Although TM doping of the $\mathrm{BaFe}_{2} \mathrm{As}_{2}$ system is convenient - providing large homogeneous crystals - it is not unique in tuning $T_{S} / T_{m}$ and $T_{c}$. Pressure can also be used to suppress $T_{S} / T_{m}$ and stabilize a low temperature superconducting state[18, 20].

In contrast with its $4 \mathrm{~d}$ neighbors $\mathrm{Rh}$ and $\mathrm{Pd}, \mathrm{Ru}$ doping provides no extra electrons to the bands. However, recent polycrystalline studies in both the $\mathrm{SrFe}_{2} \mathrm{As}_{2}\left[21\right.$, 22] and $\mathrm{BaFe}_{2} \mathrm{As}_{2}[7]$ systems show that Ru substitition on the Fe site suppresses the structural/magnetic phase transition and leads to superconductivity, indicating that this system may allow a direct comparison of nominally isovalent doping and electron doping TM substitution as well as pressure studies. Isovalent doping induced superconductivity, as pressure before it, indicates that whereas $x$ and $e$ are important parameters in parameterizing the phase transitions in these systems, changes in the unit cell parameter may be important as well.

Based on this, we have studied $\mathrm{Ru}$ doped $\mathrm{BaFe}_{2} \mathrm{As}_{2}$ single crystals in order to compare the effects of isoelectronic doping to $3 \mathrm{~d}$ and $4 \mathrm{~d}$ transition metal, electron doped compounds. 
As we wrote this work up, a similar, complimentary, study was posted; 23, 24] comparison to these data will be made as well.

\section{EXPERIMENTAL METHODS}

Single crystals of $\mathrm{Ba}\left(\mathrm{Fe}_{1-x} \mathrm{Ru}_{x}\right)_{2} \mathrm{As}_{2}$ were grown out of self flux using conventional hightemperature solution growth techniques[10, 25]. FeAs and RuAs were synthesized in the same manner as in [10]. Small Ba chunks and FeAs/RuAs powder were mixed together in a ratio of Ba:TMAs=1:4. The mixture was then placed in an alumina crucible with a "catch" crucible filled with quartz wool placed on top. Both crucibles were sealed in a silica tube under $1 / 6$ atmosphere of Ar gas. The sealed tube was heated up to $1180^{\circ} \mathrm{C}$ over 12 hours, held at $1180^{\circ} \mathrm{C}$ for 8-12 hours, and then cooled over $45-65$ hours. The final temperature varied between $1050^{\circ} \mathrm{C}$ and $1100^{\circ} \mathrm{C}$, increasing with the Ru doping level. Once the furnace reached the final temperature, the excess FeAs/RuAs liquid was decanted, leaving the single crystals behind. Unfortunately, this increasing decanting temperature made doping levels above $x=0.37$ difficult to produce.

Powder x-ray diffraction measurements, with a Si standard, were performed using a Rigaku Miniflex diffractometer with $\mathrm{Cu} K \alpha$ radiation at room temperature. Diffraction patterns were taken on ground single crystals from each batch. Only very small FeAs impurity peaks were found as a secondary phase. The unit cell parameters were refined by "Rietica" software. Elemental analysis of single crystal samples was used to determine the actual percentage of the dopant in the lattice as opposed to the nominal doping level. This was performed using wavelength dispersive x-ray spectroscopy (WDS) in a JEOL JXA8200 electron-microprobe. Magnetization data were collected in a Quantum Design (QD) Magnetic Properties Measurement System (MPMS). Temperature-dependent AC electrical resistance data $(\mathrm{f}=16 \mathrm{~Hz}, \mathrm{I}=3 \mathrm{~mA})$ was collected using either a QD MPMS with a LR700 resistance bridge or a QD Physical Properties Measurement System (PPMS). Electrical contact was made to the sample using Epotek H20E silver epoxy to attach Pt wires in a four-probe configuration. 


\section{RESULTS}

A summary of the WDS measurement data is presented in Table I. For each batch, between 1 and 5 crystal surfaces were measured. The table shows the number of points measured, the nominal $x$ value measured, the average $x$ value, and two times the standard deviation of the $x$ values measured. All $x$ values given in this paper are the average $x_{W D S}$ values determined by wavelength dispersive x-ray spectroscopy (WDS). Fig. 1 shows the measured vs nominal $\mathrm{Ru}$ concentration, as well as the error bars on the measured values. For $x_{W D S} \leq 0.21$ the variation in $\mathrm{Ru}$ content within a batch is small, in the range of $1-5 \%$ of the $x$ value. Such variation is similar to what is found for other $3 \mathrm{~d}$ and $4 \mathrm{~d}$ doping series [6, 10, 14, 17]. For $x \geq 0.24$ there is a sudden and rather dramatic increase in the variation of the $\mathrm{Ru}$ concentration within a single batch (and even a single sample). It is not clear what the origin of the change in homogeneity is, but it is also noted, in a qualitative manner, in ref.[23] as well.

\begin{tabular}{c|c|c|c|c|c|c|c|c|c|c|c}
\hline \hline \multicolumn{10}{c}{$\mathrm{Ba}\left(\mathrm{Fe}_{1-x} \mathrm{Ru}_{x}\right)_{2} \mathrm{As}_{2}$} \\
\hline $\mathrm{N}$ & 14 & 16 & 12 & 12 & 11 & 19 & 18 & 13 & 14 & 15 & 25 \\
\hline$x_{\text {nominal }}$ & 0.05 & 0.1 & 0.125 & 0.15 & 0.175 & 0.2 & 0.225 & 0.25 & 0.265 & 0.27 & 0.3 \\
\hline$x_{W D S}$ & 0.021 & 0.048 & 0.073 & 0.092 & 0.126 & 0.161 & 0.210 & 0.24 & 0.29 & 0.29 & 0.36 \\
\hline $2 \sigma$ & 0.001 & 0.001 & 0.001 & 0.003 & 0.003 & 0.005 & 0.013 & 0.05 & 0.05 & 0.05 & 0.05 \\
\hline \hline
\end{tabular}

TABLE I. WDS data for $\mathrm{Ba}\left(\mathrm{Fe}_{1-x} \mathrm{Ru}_{x}\right)_{2} \mathrm{As}_{2}$. $\mathrm{N}$ is the number of points measured in each batch, $x_{W D S}$ is the average $x$ value for that batch, and $2 \sigma$ is twice the standard deviation of the N values measured.

Powder x-ray diffraction measurements confirm that $\mathrm{Ba}\left(\mathrm{Fe}_{1-x} \mathrm{Ru}_{x}\right)_{2} \mathrm{As}_{2}$ forms in the I4/mmm, $\mathrm{ThCr}_{2} \mathrm{Si}_{2}$ structure and that impurities are minimal (Fig. 2). Rietveld refinement of the XRD data gives the $a$ and $c$ lattice parameters, which are plotted, along with the unit cell volume, as a function of $x_{W D S}$ in Fig. 3 .

Figure 4 shows the normalized electrical resistance data of the $\mathrm{Ba}\left(\mathrm{Fe}_{1-x} \mathrm{Ru}_{x}\right)_{2} \mathrm{As}_{2}$ series from $5 \mathrm{~K}$ to $300 \mathrm{~K}$. Normalized resistance is plotted instead of resistivity because of the 


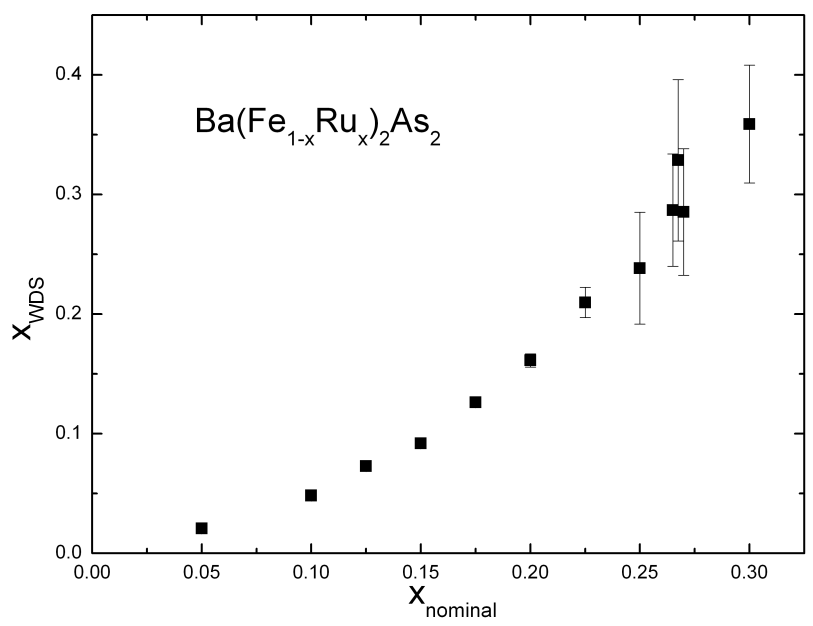

FIG. 1. Experimentally determined Ru concentration, $x_{W D S}$, vs nominal Ru concentration. Error bars are $\pm 2 \sigma$ (values from Table I).

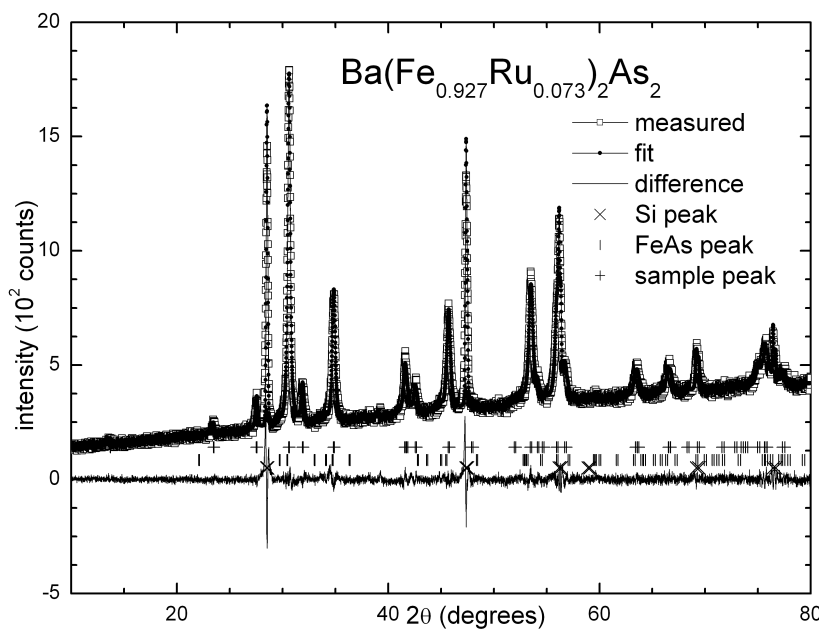

FIG. 2. Powder x-ray pattern for $\mathrm{Ba}\left(\mathrm{Fe}_{1-x} \mathrm{Ru}_{x}\right)_{2} \mathrm{As}_{2}, x=0.073$, with Si standard. Open symbols are measured data, closed ones are fit, the line shows the difference. $\times, \mid$ and + symbols are calculated peak positions for $\mathrm{Si}, \mathrm{FeAs}$ and the sample.

tendency of these samples to exfoliate or $\operatorname{crack}[10,26,27]$. The anomaly in normalized resistance at $134 \mathrm{~K}$ for pure $\mathrm{BaFe}_{2} \mathrm{As}_{2}$ is associated with a first order phase transition into an orthorhombic antiferromagnetic state[28]. As in the case of $\mathrm{Co}, \mathrm{Ni}, \mathrm{Cu}, \mathrm{Rh}$ and $\mathrm{Pd}$ substitution [6, 10, 14, 17, the temperature of the resistive anomaly is suppressed monotonically and the shape is changed from the sharp loss of resistance on cooling through $T_{S} / T_{m}$ seen in pure $\mathrm{BaFe}_{2} \mathrm{As}_{2}$ to a broader increase in resistance on cooling through $T_{S} / T_{m}$ for 


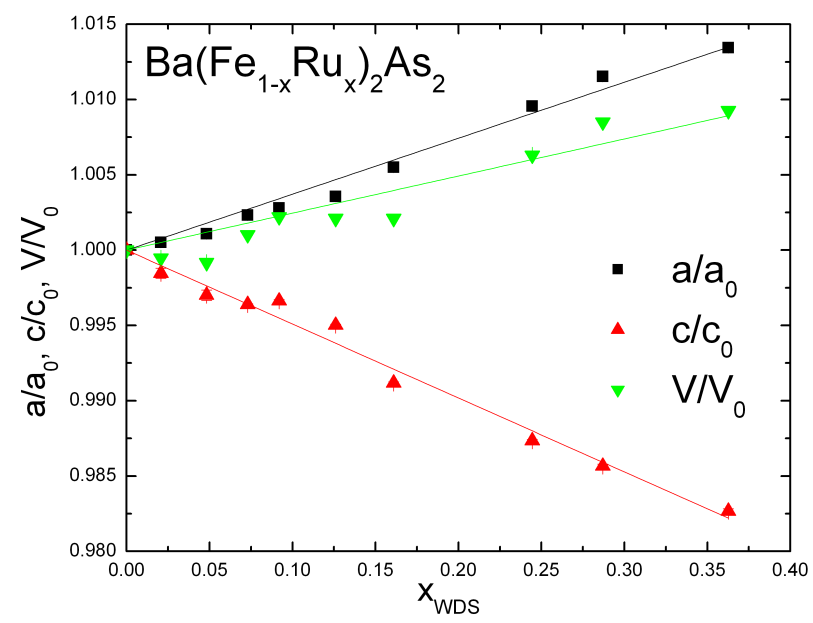

FIG. 3. Lattice parameters for $\mathrm{Ba}\left(\mathrm{Fe}_{1-x} \mathrm{Ru}_{x}\right)_{2} \mathrm{As}_{2}$, compared to $\mathrm{BaFe}_{2} \mathrm{As}_{2}$, for which $a_{0}=3.96 \AA$, $c_{0}=13.0 \AA$ and $V_{0}=204 \AA^{3}$. The slopes are $a / a_{0}:(3.7 \pm 0.1) \times 10^{-4} / \mathrm{Ru}$ atom, $c / c_{0}:(-4.9 \pm$ $0.1) \times 10^{-4} / \mathrm{Ru}$ atom, $V / V_{0}:(2.4 \pm 0.2) \times 10^{-4} / \mathrm{Ru}$ atom. The trend lines are determined by a least squares fit. The error in the slope is the standard error from this fit. (Color online)

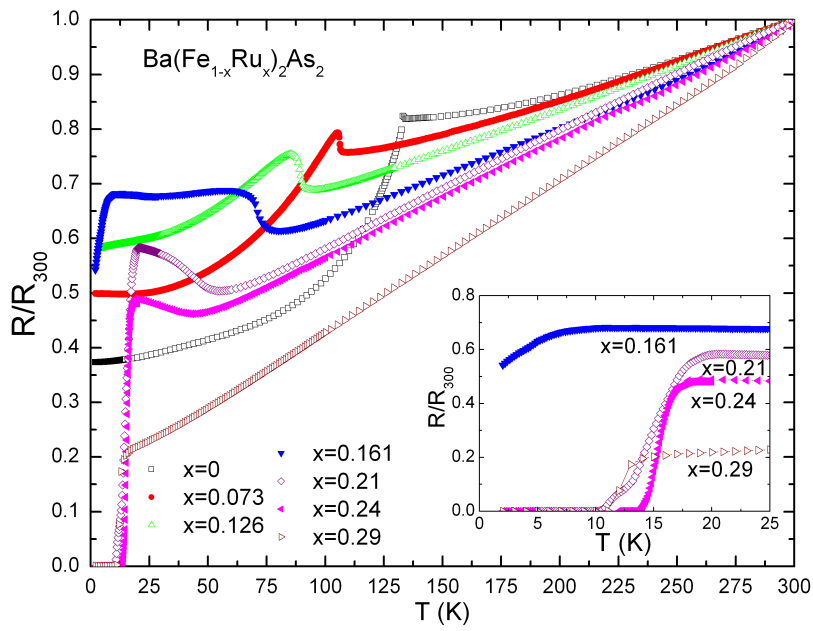

FIG. 4. Temperature dependent resistance, normalized to the room temperature value, for select $\mathrm{Ba}\left(\mathrm{Fe}_{1-x} \mathrm{Ru}_{x}\right)_{2} \mathrm{As}_{2}$ doping levels. Inset shows low temperature behavior. (Color online)

intermediate $x$ values. For $x \geq 0.29$, anomalies associated with $T_{S} / T_{m}$ are no longer detectable. Superconductivity begins to appear above $x=0.161$ (resistive onset only) and is fully manifested $(R=0)$ by $x=0.210$. A maximum $T_{c}$ of $16.5 \mathrm{~K}$ is achieved at $x \approx 0.29$. $T_{c}$ is suppressed for higher values of $x$. The superconducting transition is quite broad compared 
to other TM dopings: more than $7 \mathrm{~K}$ wide for $x_{W D S}=0.210$ compared with a $3 \mathrm{~K}$ width for a Co doping level of $x=0.038[10]$. Such a wide transition is more typical of pressure induced superconductivity rather than chemical doping[20].
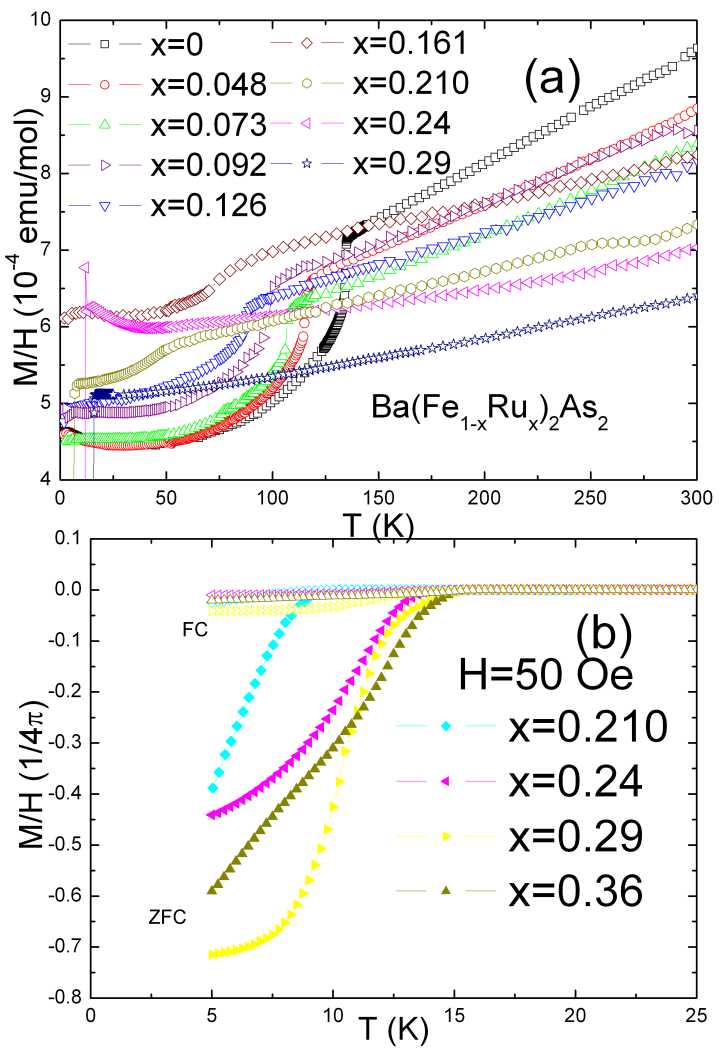

FIG. 5. (a) Temperature dependent magnetization, scaled by applied field $H=70 \mathrm{kOe}$, for $\mathrm{Ba}\left(\mathrm{Fe}_{1-x} \mathrm{Ru}_{x}\right)_{2} \mathrm{As}_{2}$. (b) Low temperature, low field, zero field cooled and field cooled magnetization for several superconducting members of the $\mathrm{Ba}\left(\mathrm{Fe}_{1-x} \mathrm{Ru}_{x}\right)_{2} \mathrm{As}_{2}$ family. $H \perp c$ for all data sets. The relatively large, low temperature, diamagnetic shielding in the zero field cooled measurements approaches that found for $\mathrm{Co}, \mathrm{Ni}, \mathrm{Rh}$ and $\mathrm{Pd}$ doping [6, 10, 14, 17].

Figure 5(a) shows high field $(\mathrm{H}=70 \mathrm{kOe}) M / H$ data for representative members of the $\mathrm{Ba}\left(\mathrm{Fe}_{1-x} \mathrm{Ru}_{x}\right)_{2} \mathrm{As}_{2}$ series. At high temperatures the $M(T) / H$ ratio is roughly linear and decreases with decreasing temperature, with a slope that decreases with increasing $\mathrm{Ru}$ doping. As with normalized resistance, the magnetization of the parent compound manifests a clear change at $134 \mathrm{~K}$, correlated with the structural/magnetic phase transition[28]. As $x$ is increased up to $x=0.126$, this transition is suppressed and broadened without qualitative change. Starting with $x=0.161$ the transition becomes much flatter and broader, and by 
$x=0.24$ it is barely visible. At $x=0.29$ it has completely vanished.

Figure 5(b) shows the low field (50 Oe) $M / H$ data for the superconducting members of the $\mathrm{Ba}\left(\mathrm{Fe}_{1-x} \mathrm{Ru}_{x}\right)_{2} \mathrm{As}_{2}$ series. These samples show a clear diamagnetic signal in the zero field cooled (ZFC) data, as well as some Meissner expulsion. It is worth noting that whereas the ZFC diamagnetic signal for $\mathrm{Co}, \mathrm{Ni}, \mathrm{Rh}, \mathrm{Pd}$ and $\mathrm{Cu} / \mathrm{Co}$ dopings are all similar and close to $-1 / 4 \pi[6,10,14,17,29]$, the low temperature values for Ru doping (5)(b)) are smaller in amplitude and vary more.

\section{DISCUSSION}

Figures 6 and 7 show normalized resistance and magnetization data, along with their derivatives, for $x=0.073$ and $x=0.16$ samples respectively. These figures show the criteria used for determining the structural/magnetic phase transition temperatures for these materials.

Figures 6(c) and 7)(c) show comparisons of normalized resistance derivatives for $\mathrm{Ru}$, Co and $\mathrm{Rh}$ doped $\mathrm{BaFe}_{2} \mathrm{As}_{2}$ with similar $T_{S} / T_{m}$ values. In the Co and Rh series, a clear splitting of the two transitions is visible. (At the same temperatures, the derivatives of magnetization and heat capacity show split features as well[6, 10, 14, 17].) By contrast, we do not see these separated features in the derivatives of the normalized resistance from the Ru system. These features have been shown to correspond to a splitting of the joint transition into two transitions, one structural the other magnetic [6, 14, 16, 17, 30]. Although the authors of ref. 23] claim to see a split transition, it appears to be a subtle feature compared to Co or $\mathrm{Rh}$ data. The single feature in the $\mathrm{Ru}$ doped series $d R / d T$ data suggests that either the splitting is much smaller, or absent, in this system or that the resistive feature associated with $T_{S}$ is much weaker in this system. It is possible that the splitting is caused by the extra electrons provided by other TM doping (eg. Co, Ni, Cu, Rh, Pd).

Onset and offset criteria were used to determine $T_{c}$ from this resistance data. $T_{c}$ was determined from the magnetization data by extrapolating the maximum slope of the ZFC data back to the normal state. There is fair agreement between $T_{c}^{\text {offset }}$ determined from normalized resistance and $T_{c}$ determined from magnetization. It should be noted, though,

that (i) superconductivity primarily occurs in the region where the spread in $x_{W D S}$ is large, and (ii) the superconducting transition is broad in $R(T)$ and both ZFC and field cooled 

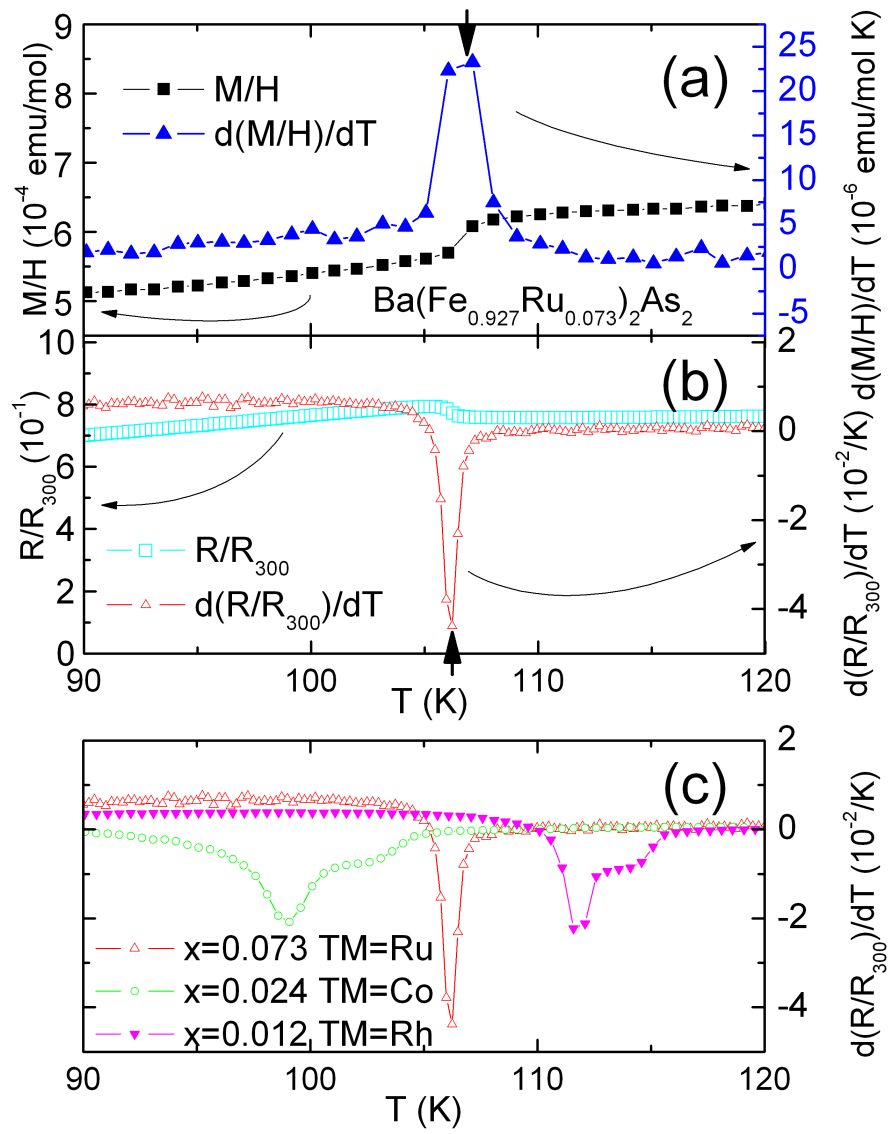

FIG. 6. Magnetization (a) and normalized resistance (b), along with derivatives, for $\mathrm{Ba}\left(\mathrm{Fe}_{1-x} \mathrm{Ru}_{x}\right)_{2} \mathrm{As}_{2}(x=0.073)$. Vertical arrows show the criteria for determination of the transition temperature. (c) shows normalized resistance derivative data for Co doping $(\mathrm{x}=0.024)$ and Rh doping $(\mathrm{x}=0.012)$ with similar transition temperatures. (Color online)

Meissner data are somewhat lower than for other TM doped series.

Using these criteria, the data presented in Figs. 4 and 5 are summarized in a $T-x$ phase diagram shown in Fig. 8. Overall, the phase diagram for the $\mathrm{Ba}\left(\mathrm{Fe}_{1-x} \mathrm{Ru}_{x}\right)_{2} \mathrm{As}_{2}$ series is qualitatively quite similar to that of the $\mathrm{Co}, \mathrm{Ni}, \mathrm{Rh}$ and $\mathrm{Pd}$ diagrams: increasing $x$ suppresses the structural/magnetic phase transition, a superconducting dome appears above some critical $x$ value, and this dome has a maximum near the point where $T_{S} / T_{m}$ extrapolates to zero. However, there is a key difference: suppression of $T_{S} / T_{m}$ is much slower than for other TM dopings (Co, Ni, $\mathrm{Cu}, \mathrm{Rh}, \mathrm{Pd})[\underline{6}$, 10, 14, 17, 29]. In previous comparisons of $3 \mathrm{~d}$ and $4 \mathrm{~d}$ TM dopings [6, 14, 17, we showed that suppression of $T_{S} / T_{m}$ 


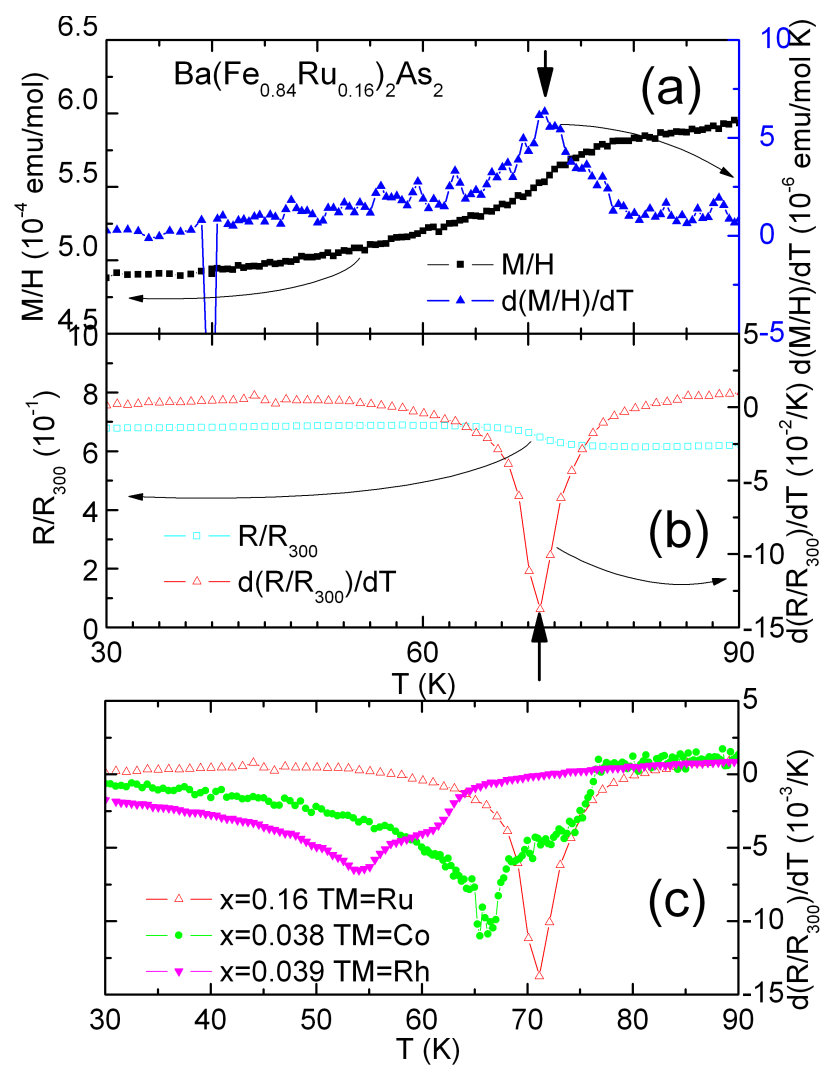

FIG. 7. Magnetization (a) and normalized resistance (b), along with derivatives, for $\mathrm{Ba}\left(\mathrm{Fe}_{1-x} \mathrm{Ru}_{x}\right)_{2} \mathrm{As}_{2}(x=0.16)$. Vertical arrows show the criteria for determination of the transition temperature. (c) shows normalized resistance derivative data for Co doping $(\mathrm{x}=0.038)$ and Rh doping $(\mathrm{x}=0.039)$ with similar transition temperatures. (Color online)

occurs at roughly the same rate regardless of differences in size and electron count between dopants; the suppression of $T_{S} / T_{m}$ in $\mathrm{Ba}\left(\mathrm{Fe}_{1-x} \mathrm{Ru}_{x}\right)_{2} \mathrm{As}_{2}$ is about three times slower.

As in the case of $\mathrm{Rh}$ and $\mathrm{Pd}$ doped $\mathrm{BaFe}_{2} \mathrm{As}_{2}[6]$, with $\mathrm{Ru}$ doping the $c$-lattice parameter shrinks compared to the parent $\mathrm{BaFe}_{2} \mathrm{As}_{2}$, while the $a$-lattice parameter and the unit cell volume, V, grow. (This is in contrast to the 3d TM dopings, where all three shrink with increasing $x$.) By way of comparison: a Ru doping level of $x_{W D S}=0.175$ has $a=1.002 a_{0}$ and $c=0.995 c_{0}$ and a Rh doping level of $x=0.171$ has $a=1.007 a_{0}$ and $c=0.988 c_{0} \cdot 6$. Because the crystallographic trends of all three 4d TM dopant series ( $\mathrm{Ru}, \mathrm{Rh}$ and $\mathrm{Pd})$ are similar, the major differences in their $T-x$ phase diagrams suggest that steric effects alone are not enough to explain the differences in behavior of this system with doping (ie. the extra electrons in $\mathrm{Rh}$ and $\mathrm{Pd}$ are responsible for the much more rapid effects of doping). 


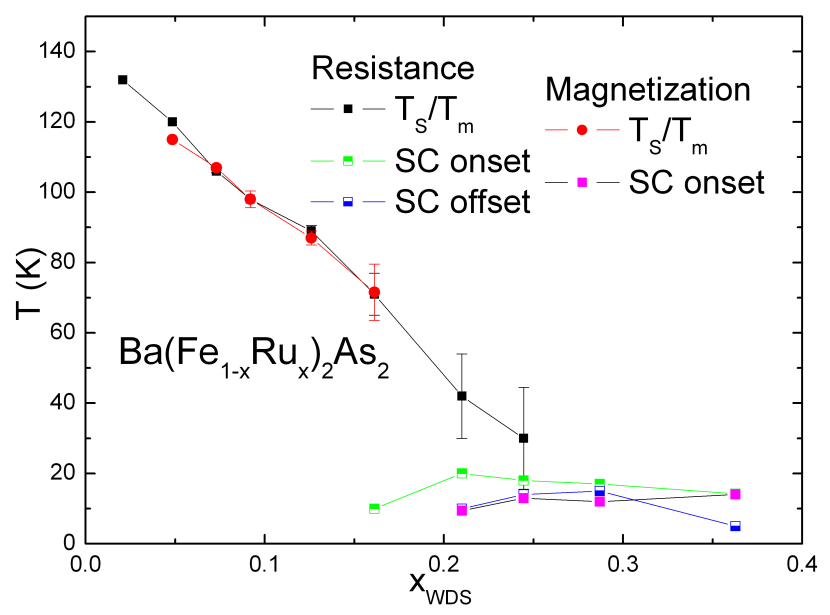

FIG. 8. $x$ dependent phase diagram, showing $T$ for salient features in $\mathrm{Ba}\left(\mathrm{Fe}_{1-x} \mathrm{Ru}_{x}\right)_{2} \mathrm{As}_{2}$. $(\mathrm{Color}$ online)

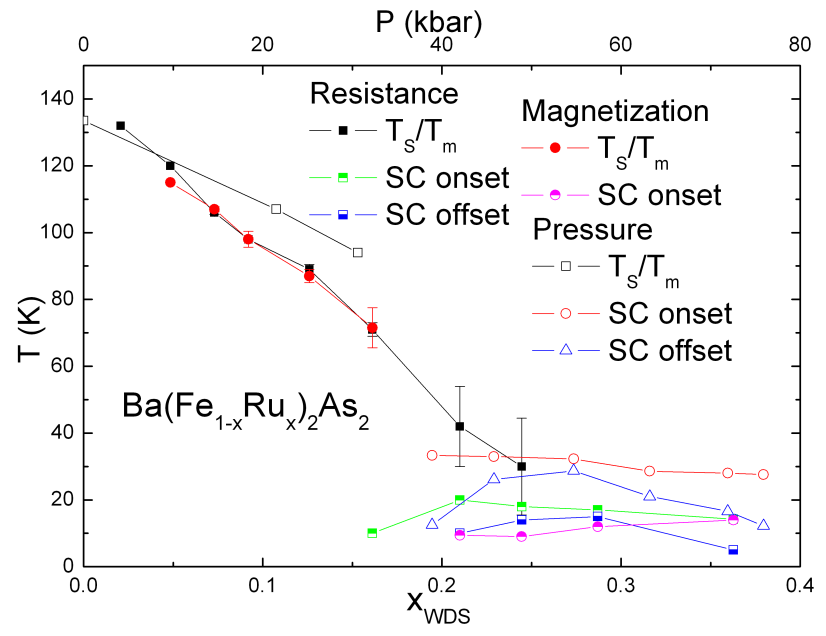

FIG. 9. Comparison of $\mathrm{Ru}$ doping phase diagram with that of the parent $\mathrm{BaFe}_{2} \mathrm{As}_{2}$ compound under applied pressure. (Color online)

Although the maximum superconducting critical temperature, $T_{c}^{\max }$, is significantly lower in the $\mathrm{Ru}$ doped system, there is a clear similarity between the $\mathrm{Ru}$ doped $T-x$ phase diagram and the pressure dependent, $T-p$, phase diagram of the parent $\mathrm{BaFe}_{2} \mathrm{As}_{2}$ compound[20], as can be seen in Fig. 9, The similarity of the phase diagrams suggests that changes in the unit cell dimensions may be playing a large role in determining the superconducting behavior, with the effects of $\mathrm{Ru}$ substituion in this system being similar to physical pressure in the 

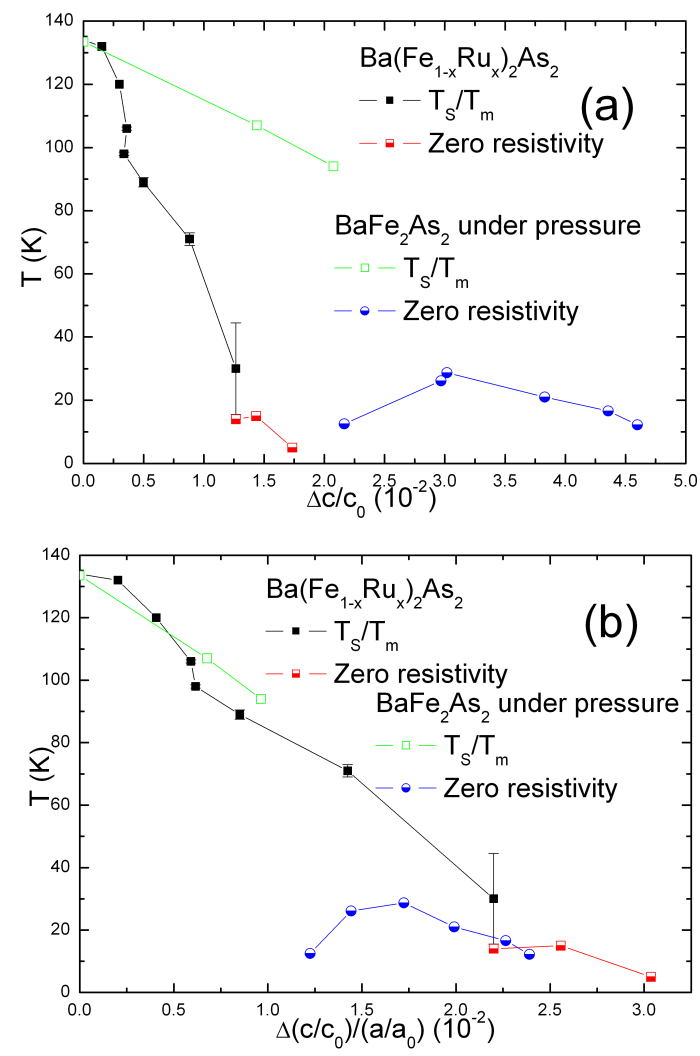

FIG. 10. Phase diagrams of $\mathrm{Ba}\left(\mathrm{Fe}_{1-x} \mathrm{Ru}_{x}\right)_{2} \mathrm{As}_{2}$ and of parent $\mathrm{BaFe}_{2} \mathrm{As}_{2}$ under pressure, scaled by lattice parameters. (a) is scaled by $\Delta c / c_{0}$. (b) is scaled by $\Delta\left(c / c_{0}\right) /\left(a / a_{0}\right)$. (Color online)

undoped $\mathrm{BaFe}_{2} \mathrm{As}_{2}$ system. The difference in $T_{c}^{\max }$ is most likely caused by the $\mathrm{Ru}$ dopant disordering the Fe-plane, whereas pressure induces no such distortion.

Whereas the agreement between the $T-x$ and $T-p$ phase diagrams in Fig. 9 is good, the scaling between $x$ and $p$ was arbitrarily choosen to optimize the overlap of the two data sets. Using our data on the $x$-dependence of the unit cell parameters (3) in combination with the data from ref. [31] on the pressure dependence of the unit cell parameters of $\mathrm{BaFe}_{2} \mathrm{As}_{2}$, we can make this comparison more quantitative. Of the four combinations of the unit cell parameters: $a, c, \mathrm{~V}$ and $c / a$, only $c$ and $c / a$ show similar responses to pressure and doping; $a$ and $\mathrm{V}$ both increase with doping whereas they decrease with $p$. Figures 10 (a) and (b) present our Ru-doping data as well as the pressure data from ref. [20] plotted as functions of the changes in $c$ and $c / a$. A comparison of these two figures clearly indicates that $c / a$ rather than $c$ better parameterizes the effects of doping and pressure. This result means that, based on these two isoelectronic perturbations (pressure and Ru doping), changes in 
the $c / a$ ratio appear to be more physically important than changes in $c$ alone.

The other isoelectronic substitution which produces superconductivity in $\mathrm{BaFe}_{2} \mathrm{As}_{2}$ is $\mathrm{P}$ doping on the As site 32 , 33]. Although the maximum $T_{c}$ in the $\mathrm{BaFe}_{2}\left(\mathrm{As}_{1-x} \mathrm{P}_{x}\right)_{2}$ system is quite a bit higher than in the $\mathrm{Ba}\left(\mathrm{Fe}_{1-x} \mathrm{Ru}_{x}\right)_{2} \mathrm{As}_{2}$ system $(\sim 30 \mathrm{~K})$, several key properties are similar. $T_{S} / T_{m}$ is suppressed in a relatively gradual manner and the maximum $T_{c}$ value occurs at a comparably high doping level $\left(x_{R u}=0.29, x_{P}=0.32\right)$ and extends over a much wider range than in any of the electron doped TM series 32, 33. Furthermore, both Ru doping and underdoping of $\mathrm{P}$ produce wider transitions than other TM dopings (eg. Co, $\mathrm{Ni}, \mathrm{Rh}, \mathrm{Pd})[6,10,33$, 34]. On the other hand, taking changes in $c$ and $a$ with $\mathrm{P}$ doping into account, $T_{S} / T_{m}$ and $T_{c}$ for $\mathrm{P}$-doped and $\mathrm{Ru}$-doped $\mathrm{BaFe}_{2} \mathrm{As}_{2}$ scale better with changes in $c$ than with changes in $c / a[33]$. This means that, if we include P-doping as a third isoelectronic perturbation, then neither changes in $c$ nor $c / a$ universally describe the $T-x$ and $T-p$ phase diagrams.

\section{SUMMARY}

Single crystals of $\mathrm{Ba}\left(\mathrm{Fe}_{1-x} \mathrm{Ru}_{x}\right)_{2} \mathrm{As}_{2}$ can be grown for $x<0.37$, although Ru homogeneity becomes less well controlled for $x>0.21$. The structural and magnetic phase transition temperature, $T_{S} / T_{m}$, is suppressed as $x$ increases but does not clearly split, as it does for $\mathrm{TM}=\mathrm{Co}, \mathrm{Ni}, \mathrm{Cu}, \mathrm{Rh}$, and $\mathrm{Pd}$ doping. As $T_{S} / T_{m}$ is suppressed superconductivity appears, reaching a maximum $T_{c}$ value of $16.5 \mathrm{~K}$ for $x=0.29$, near the point that $T_{S} / T_{m}$ extrapolates to $T=0 \mathrm{~K}$. Whereas the suppression of $T_{S} / T_{m}$ and the stabilization of $T_{c}$ occur at a much slower rate for $\mathrm{Ru}$ doping than they do for doping with $\mathrm{TM}=\mathrm{Co}, \mathrm{Ni}, \mathrm{Cu}$, $\mathrm{Rh}$, or $\mathrm{Pd}$, indicating that the additional electrons brought by these dopants play a significant role in tuning of this system, there is a remarkable agreement between two isoelectronic phase diagrams (Ru-doping and pressure) of $\mathrm{BaFe}_{2} \mathrm{As}_{2}$ when plotted as $T(c / a)$, but not when plotted as $T(c)$.

\section{ACKNOWLEDGEMENTS}

Work at the Ames Laboratory was supported by the Department of Energy, Basic Energy Sciences under Contract No. DE-AC02-07CH11358. We would like to thank E. D. Mun, S. 
Kim, X. Lin, R. Gordon, R. Fernandes, A. Kreyssig, R. Roggers and J. Brgoch for help and useful discussions.

[1] Y. Kamihara, T. Watanabe, M. Hirano, and H. Hosono, Journal of the American Chemical Society, 130, 3297 (2008).

[2] M. Rotter, M. Tegel, and D. Johrendt, Phys. Rev. Lett., 101, 107006 (2008).

[3] R. Zhi-An, L. Wei, Y. Jie, Y. Wei, S. Xiao-Li, Zheng-Cai, C. Guang-Can, D. Xiao-Li, S. LiLing, Z. Fang, and Z. Zhong-Xian, Chinese Physics Letters, 25, 2215 (2008).

[4] A. S. Sefat, R. Jin, M. A. McGuire, B. C. Sales, D. J. Singh, and D. Mandrus, Phys. Rev. Lett., 101, 117004 (2008).

[5] A. S. Sefat, A. Huq, M. A. McGuire, R. Jin, B. C. Sales, D. Mandrus, L. M. D. Cranswick, P. W. Stephens, and K. H. Stone, Phys. Rev. B, 78, 104505 (2008).

[6] N. Ni, A. Thaler, A. Kracher, J. Q. Yan, S. L. Bud'ko, and P. C. Canfield, Physical Review B (Condensed Matter and Materials Physics), 80, 024511 (2009).

[7] S. Sharma, A. Bharathi, S. Chandra, V. R. Reddy, S. Paulraj, A. T. Satya, V. S. Sastry, A. Gupta, and C. S. Sundar, Phys. Rev. B, 81, 174512 (2010).

[8] X. L. Wang, H. Y. Shi, X. W. Yan, Y. C. Yuan, Z.-Y. Lu, X. Q. Wang, and T.-S. Zhao, Applied Physics Letters, 96, 012507 (2010).

[9] S. R. Saha, T. Drye, K. Kirshenbaum, N. P. Butch, P. Y. Zavalij, and J. Paglione, Journal of Physics: Condensed Matter, 22, 072204 (2010).

[10] N. Ni, M. E. Tillman, J.-Q. Yan, A. Kracher, S. T. Hannahs, S. L. Bud'ko, and P. C. Canfield, Physical Review B (Condensed Matter and Materials Physics), 78, 214515 (2008).

[11] J.-H. Chu, J. G. Analytis, C. Kucharczyk, and I. R. Fisher, Physical Review B (Condensed Matter and Materials Physics), 79, 014506 (2009).

[12] F. Ning, K. Ahilan, T. Imai, A. S. Sefat, R. Jin, M. A. McGuire, B. C. Sales, and D. Mandrus, Journal of the Physical Society of Japan, 78, 013711 (2009).

[13] L. Fang, H. Luo, P. Cheng, Z. Wang, Y. Jia, G. Mu, B. Shen, I. I. Mazin, L. Shan, C. Ren, and H.-H. Wen, Phys. Rev. B, 80, 140508 (2009).

[14] P. C. Canfield, S. L. Bud'ko, N. Ni, J. Q. Yan, and A. Kracher, Phys. Rev. B, 80, 060501 (2009). 
[15] C. Lester, J.-H. Chu, J. G. Analytis, S. C. Capelli, A. S. Erickson, C. L. Condron, M. F. Toney, I. R. Fisher, and S. M. Hayden, Physical Review B (Condensed Matter and Materials Physics), 79, 144523 (2009).

[16] D. K. Pratt, W. Tian, A. Kreyssig, J. L. Zarestky, S. Nandi, N. Ni, S. L. Bud'ko, P. C. Canfield, A. I. Goldman, and R. J. McQueeney, Phys. Rev. Lett., 103, 087001 (2009).

[17] P. C. Canfield and S. L. Bud'ko, arXiv:1002.0858v1 [cond-mat.supr-con].

[18] M. S. Torikachvili, S. L. Bud'ko, N. Ni, and P. C. Canfield, Phys. Rev. B, 78, 104527 (2008).

[19] P. L. Alireza, Y. T. C. Ko, J. Gillett, C. M. Petrone, J. M. Cole, G. G. Lonzarich, and S. E. Sebastian, Journal of Physics: Condensed Matter, 21, 012208 (2009).

[20] E. Colombier, S. L. Bud'ko, N. Ni, and P. C. Canfield, Phys. Rev. B, 79, 224518 (2009).

[21] W. Schnelle, A. Leithe-Jasper, R. Gumeniuk, U. Burkhardt, D. Kasinathan, and H. Rosner, Phys. Rev. B, 79, 214516 (2009).

[22] Y. Qi, L. Wang, Z. Gao, D. Wang, X. Zhang, and Y. Ma, Physica C: Superconductivity, 469, 1921 (2009).

[23] F. Rullier-Albenque, D. Colson, A. Forget, P. Thuéry, and S. Poissonnet, Phys. Rev. B, 81, 224503 (2010).

[24] V. Brouet, F. Rullier-Albenque, M. Marsi, B. Mansart, J. Faure, L. Perfetti, A. Taleb-Ibrahimi, P. L. Fevre, F. Bertran, A. Forget, and D. Colson, arXiv:1002.4952v1 [cond-mat.supr-con].

[25] P. C. Canfield and Z. Fisk, Phil. Mag. B, 65, 1117 (1992).

[26] M. A. Tanatar, N. Ni, C. Martin, R. T. Gordon, H. Kim, V. G. Kogan, G. D. Samolyuk, S. L. Bud'ko, P. C. Canfield, and R. Prozorov, Physical Review B (Condensed Matter and Materials Physics), 79, 094507 (2009).

[27] M. A. Tanatar, N. Ni, G. D. Samolyuk, S. L. Bud'ko, P. C. Canfield, and R. Prozorov, Physical Review B (Condensed Matter and Materials Physics), 79, 134528 (2009).

[28] M. Rotter, M. Tegel, D. Johrendt, I. Schellenberg, W. Hermes, and R. Pottgen, Physical Review B (Condensed Matter and Materials Physics), 78, 020503 (2008).

[29] N. Ni, Structural / magnetic phase transitions and superconductivity in $\mathrm{Ba}\left(\mathrm{Fe}_{1-x} \mathrm{TM}_{x}\right)_{2} A s_{2}$ $(T M=C o, N i, C u, C o / C u, R h$ and $P d)$ single crystals, Ph.D. thesis, Iowa State University (2009).

[30] A. Kreyssig, M. G. Kim, S. Nandi, D. K. Pratt, W. Tian, J. L. Zarestky, N. Ni, A. Thaler, S. L. Bud'ko, P. C. Canfield, R. J. McQueeney, and A. I. Goldman, Phys. Rev. B, 81, 134512 
$(2010)$.

[31] S. A. J. Kimber, A. Kreyssig, Y.-Z. Zhang, H. O. Jeschke, R. Valent, F. Yokaichiya, E. Colombier, J. Yan, T. C. Hansen, T. Chatterji, R. J. McQueeney, P. C. Canfield, A. I. Goldman, and D. N. Argyriou, Nature Materials, 8, 471 (2009).

[32] S. Jiang, H. Xing, G. Xuan, C. Wang, Z. Ren, C. Feng, J. Dai, Z. Xu, and G. Cao, Journal of Physics: Condensed Matter, 21, 382203 (2009).

[33] S. Kasahara, T. Shibauchi, K. Hashimoto, K. Ikada, S. Tonegawa, R. Okazaki, H. Shishido, H. Ikeda, H. Takeya, K. Hirata, T. Terashima, and Y. Matsuda, Phys. Rev. B, 81, 184519 $(2010)$.

[34] L. J. Li, Y. K. Luo, Q. B. Wang, H. Chen, Z. Ren, Q. Tao, Y. K. Li, X. Lin, M. He, Z. W. Zhu, G. H. Cao, and Z. A. Xu, New Journal of Physics, 11, 025008 (2009). 